\title{
WHAT IS SGR A*?
}

The Starved Black Hole in the Center of the Milky Way

\author{
H. FALCKE \\ Max-Planck-Institut für Radioastronomie (MPIfR) \\ Auf dem Hügel 69, D-53121 Bonn, Germany \\ E-mail: HFalcke@mpifr-bonn.mpg.de (hfalcke@astro.umd.edu)
}

\section{Introduction}

Sgr $\mathrm{A}^{*}$ is the unique $1 \mathrm{Jy}$ flat spectrum radio point source located at the dynamical center of the Galaxy and in the very center of the central star cluster (Eckart et al. 1993). Due to its unusual appearance it has long been speculated that this source is powered by a supermassive black hole - an object whose presence has been suspected to reside in the nuclei of many other galaxies as well. Its mass is believed to be as large as $M_{\bullet} \sim 2 \cdot 10^{6} M_{\odot}$ (e.g. Genzel \& Townes 1987) while a lower limit of $M_{\bullet}>200-2000 M_{\odot}$ can be inferred from the low proper motion of Sgr A* (Backer - this volume). The currently best dynamical arguments for the million solar mass black hole stem from detailed stellar kinematic studies (Haller et al. 1995).

The enormous increase in observational data obtained for $\mathrm{Sgr} \mathrm{A}^{*}$ in recent years has enabled us to develop, compare and constrain a variety of models for the emission characteristics of this source. Because of its relative proximity and further observational input to come $\mathrm{Sgr} \mathrm{A}^{*}$ may therefore become one of the best laboratories for studying supermassive black hole candidates and basic AGN physics. This paper briefly summarizes our current understanding of this enigmatic radio source.

\section{Observational Input}

\subsection{RADIO-SUBMM SPECTRUM}

The radio spectrum of $\mathrm{Sgr} \mathrm{A}^{*}$ has been extensively studied in the range 1-600 GHz where it mostly shows substantial variability. As there are only very few quasi-simultaneous flux density measurements available (see Wright \& Backer 1993) an exact description of the radio spectrum is very 
uncertain at the moment. An averaged spectrum combined of various data sets available in the literature (Duschl \& Lesch 1994) may be fitted by a single powerlaw with spectral index $\alpha \sim 1 / 3\left(S_{\nu} \propto \nu^{\alpha}\right)$. However, it appears as if the submm regime is less variable than the radio regime (Zylka et al. 1995) and there might even be a weak submm-excess (Zylka et al. 1992; compare also Rogers et al. 1994 with Zylka et al. 1995). Sgr A* is not seen at IR wavelength and hence the spectrum must cut-off towards $12 \mu \mathrm{m}$ (Zylka et al. 1992; Gezari - this volume). The spectrum also cuts off below $1 \mathrm{GHz}$.

\subsection{HIGH ENERGIES}

Evidence has grown substantially that $\mathrm{Sgr} \mathrm{A}^{*}$ is also an X-ray emitter. Art-P/GRANAT detected an x-ray source coinciding with the position of Sgr A* within $40^{\prime \prime}$ (Sunyaev et al. 1991). It shows variability within a factor 2 over a period of several months. The identification as $\mathrm{Sgr} \mathrm{A}^{*}$ is corroborated by a ROSAT detection of this source with a positional uncertainty of only 10" (Predehl \& Trümper 1994). The ROSAT flux, however, was lower than expected from the Art-P measurements and prompted the interpretation of additional intrinsic absorption in Sgr A* - if the ROSAT and the Art-P source are identical. The spectrum in the Art-P band (4-20 $\mathrm{keV}$ ) is a hard powerlaw with $\alpha \sim-0.6$ and breaks already in the range $35-100 \mathrm{keV}$ (Goldwurm et al. 1994). The situation, however, seems to have changed now, after the launch of ASCA. Koyama (1994) and Maeda et al. (1996) report that the hard source at the Galactic Center (GC) and the soft ROSAT source are offset by 1.3 arcmin. They claim that the hard souce which is most likely the source detected by Art-P - is not $\mathrm{Sgr} \mathrm{A*}$, but a transient $\mathrm{x}$-ray binary. This means that the total x-ray luminosity of Sgr $A^{*}$ could be as low as $10^{5} L_{\odot}$ and lower.

There is also a gamma-ray detection of the GC with EGRET (Mattox et al. 1992) but at present it is not clear whether this is a point source or extended emission.

\subsection{LUMINOSITY CONSTRAINTS}

The bolometric optical-UV luminosity of $\mathrm{Sgr} \mathrm{A}^{*}$ can be estimated from the fact that a luminous point source should contribute to the heating of the surrounding dust and thus be visible in submm-IR data (Falcke et al. 1993a) - which is not the case. Hence, we estimated that Sgr A* cannot be very luminous with $L_{\mathrm{UV}} \leq$ a few $10^{5} L_{\odot}$. Recently Zylka et al. (1995) have updated their submm measurements of the Sgr A region and concluded from the low temperature gradients in the dust that the dust heating can not be dominated by a single point source but is more likely 
due to a cluster of luminous stars (e.g. Krabbe et al. 1991). This would also indicate that $\mathrm{Sgr} \mathrm{A}^{*}$ has a bolometric luminosity of not more than a few $10^{5} L_{\odot}$, but this estimate could be uncertain by a factor 10 . A lower limit derived from the claimed detection of Sgr A* at NIR wavelengths (Eckart et al. 1992) has become uncertain as this source was now resolved into a cluster of stars (Genzel - this volume; Eckart et al. 1995) making it difficult to identify Sgr A* with the present uncertainties between the radio and optical reference frame. Provided the separation of the 5 sources is real, the upper limit for Sgr A* in the K band could be $L_{\nu}<3 \cdot 10^{21} \mathrm{erg} / \mathrm{sec} / \mathrm{Hz}$. In the IR Gezari (this volume) has reported an upper limit at $12.4 \mu \mathrm{m}$ of $0.9 \cdot 10^{22} \mathrm{erg} / \mathrm{sec} / \mathrm{Hz}$. With all these upper limits one starts to wonder whether $\mathrm{Sgr} \mathrm{A}^{*}$ is actually there, and without the compact radio source we would have not the slightest clue that there is something. However, we have argued earlier that in order to explain the radio emission, one needs $L_{\mathrm{UV}}>10^{4} L_{\odot}$ (Falcke et al. 1993b) and there is still hope that finally $\mathrm{Sgr}$ $\mathrm{A}^{*}$ will reveal itself unambiguously at other wavelengths as well.

\subsection{SOURCE SIZE}

The mm-submm size of $\operatorname{Sgr~A*}$ is constrained at least within one order of magnitude. From the absence of refractive scintillation Gwinn et al. (1991) have argued that $\mathrm{Sgr} \mathrm{A}^{*}$ must be larger than $10^{12} \mathrm{~cm}$ at $\lambda 1.3$ and $\lambda 0.8$ $\mathrm{mm}$. Krichbaum et al. (1993 \& 1994) obtained source sizes for Sgr $\mathrm{A}^{*}$ of $4.2 \cdot 10^{13} \mathrm{~cm}$ at $86 \mathrm{GHz}$ and $9.5 \cdot 10^{13} \mathrm{~cm}$ at $43 \mathrm{GHz}$ with VLBI - the latter well above the expected scattering size as extrapolated from lower frequencies. This claim is challenged by Rogers et al. (1994) who only get $2 \cdot 10^{13} \mathrm{~cm}$ at $86 \mathrm{GHz}$ in an experiment with a factor 2 shorter baseline. Krichbaum et al. (1993) also found additional weak components and a somewhat elongated source structure at $43 \mathrm{GHz}$ VLBI not seen by Backer et al. (1993). A possibility to reconcile the results could be source variability and elongation of the internal structure which would lead to different sizes if observed with differently oriented baselines. It will be very interesting to see the results of further mm-VLBI experiments.

\section{Properties of the Radio Source}

\subsection{A HOMOGENOUS BLOB?}

Recent submm measurements (see Zylka et al. 1995) indicate that the radio spectrum of Sgr A* continues up to several hundred $\mathrm{GHz}$ with peak fluxes around 3.5 Jy and a sharp cut-off towards the IR. The submm spectrum can no longer be explained by thermal dust emission as this would require extremely cold dust $(\sim 15 \mathrm{~K})$ which is very unlikely because of the intense 
(stellar) radiation field in the Galactic Center. To explain the flat submm spectrum with synchrotron emission one needs either a combination of selfabsorbed components (requiring high compactness) or an electron distribution where the bulk of the electron energy is concentrated in a narrow energy interval. The latter could be either a very flat electron powerlaw distribution $\left(d N / d \gamma \propto \gamma^{-p}\right)$ with $p<1 / 3$ and sharp high-energy cut-off, a steep powerlaw with low-energy cut-off, a monoenergetic (e.g. an electron beam) or a thermal distribution.

Duschl \& Lesch (1994, also this volume) suggested that the radio emission of $\mathrm{Sgr} \mathrm{A}^{*}$ can simply be explained with a single homogenous blob of monoenergetic electrons. Although this cannot be quite true in its most rigorous formulation, as argued below, one can use this approach to get a fairly good idea of the basic parameters of the Sgr A* radio source: the required model parameters are the magnetic field $B$, the Lorentz factor $\gamma_{e}$, the electron density $n_{\mathrm{e}}$, the volume $V=\pi R^{2} Z$ (assumed to be cylindric) and the distance set to $8.5 \mathrm{kpc}$. On the other side we have three measurable input parameters: the peak frequency $\nu_{\max } \sim \nu_{\mathrm{c}} / 3.5$ of a monoenergetic synchrotron spectrum, the peak flux $S_{\nu_{\max }}$ and the VLBI source size (see above). A fourth parameter can be gained if one assumes that magnetic field and relativistic electrons are in equipartition, i.e. $B^{2} / 8 \pi=k n_{\mathrm{e}} \gamma_{\mathrm{e}} m_{\mathrm{e}} c^{2}$ with $k \sim 1$. With this condition we obtain (averaged over pitch angle) that

$$
\gamma_{\mathrm{e}}=326 k^{1 / 7}\left(\frac{F_{\nu_{\max }}}{3.5 \mathrm{Jy}}\right)^{-1 / 7}\left(\frac{\nu_{\max }}{10^{12} \mathrm{~Hz}}\right)^{3 / 7}\left(\frac{R}{10^{13} \mathrm{~cm}}\right)^{2 / 7}\left(\frac{Z}{4 \cdot 10^{13} \mathrm{~cm}}\right)^{1 / 7}
$$

$B=10 \mathrm{G} k^{-2 / 7}\left(\frac{F_{\nu_{\max }}}{3.5 \mathrm{Jy}}\right)^{2 / 7}\left(\frac{\nu_{\max }}{10^{12} \mathrm{~Hz}}\right)^{1 / 7}\left(\frac{R}{10^{13} \mathrm{~cm}}\right)^{-4 / 7}\left(\frac{Z}{4 \cdot 10^{13} \mathrm{~cm}}\right)^{-2 / 7}$

$n_{\mathrm{e}}=\frac{1.4 \cdot 10^{4}}{\mathrm{~cm}^{3}} k^{2 / 7}\left(\frac{F_{\nu_{\max }}}{3.5 \mathrm{Jy}}\right)^{5 / 7}\left(\frac{\nu_{\max }}{10^{12} \mathrm{~Hz}}\right)^{-1 / 7}\left(\frac{R}{10^{13} \mathrm{~cm}}\right)^{-10 / 7}\left(\frac{Z}{4 \cdot 10^{13} \mathrm{~cm}}\right)^{-5 / 7}$.

Apparently the 'non'-equipartition parameter $k$ enters only weakly and as long as one is not very far from equipartition the parameters are basically fixed: $\nu_{\max }$ is known within a factor three, $S_{\nu_{\max }}$ within $50 \%$ and the source size within a factor 10 . This means that models advocating very high electron Lorentz factors $\left(\gamma_{\mathrm{e}} \sim 10^{4}\right.$, Kundt 1990) deviate from equipartition by $\sim 10$ orders of magnitude!

Because of the high compactness of Sgr A* synchrotron self-absorption becomes another important point to be considered. Using an absorption 
coefficient of $\kappa_{\text {sync }}=1.4 \cdot 10^{-9} \mathrm{~cm}^{-1}\left(n_{\mathrm{e}} / \mathrm{cm}^{-3}\right)(B / \mathrm{G}) \gamma_{\mathrm{e}}^{-5}\left(\nu / \nu_{\mathrm{c}}\right)^{-5 / 3}$ one finds the synchrotron self-absorption frequency to be

$\nu_{\mathrm{ssa}}=\frac{2.5 \mathrm{GHz}}{k^{0.09}}\left(\frac{F_{\nu_{\max }}}{3.5 \mathrm{Jy}}\right)^{0.69}\left(\frac{\nu_{\max }}{10^{12} \mathrm{~Hz}}\right)^{-.46}\left(\frac{R}{10^{13} \mathrm{~cm}}\right)^{-.77}\left(\frac{Z}{4 \cdot 10^{13} \mathrm{~cm}}\right)^{-.69}$.

Here we took the maximum sizes allowed by mm-VLBI; if further studies show that $\mathrm{Sgr} \mathrm{A} \mathrm{A}^{*}$ is even more compact at submm then $\nu_{\text {ssa }}$ will increase further making it completely impossible to describe the whole spectrum with a single component.

\subsection{SUBMM SOURCE SIZE}

We can now make very solid arguments about the possible source size of Sgr $\mathrm{A}^{*}$ at submm wavelengths. As VLBI measurements are only available at higher wavelengths one could still postulate arbitrarily large submm source sizes. However, if $\mathrm{Sgr} \mathrm{A}$ (submm) were optically thin and larger than $4 \cdot 10^{13}$ $\mathrm{cm}$ we should have seen the low frequency $\nu^{1 / 3}$ part of its spectrum with $3 \mathrm{~mm}$ VLBI already. This could only be avoided if the submm component becomes optically thick below $\sim 100 \mathrm{GHz}$. As shown above this is possible only for a very compact source where the dimensions of $\mathrm{Sgr} \mathrm{A}^{*}$ at submm wavelengths are substantially smaller than at $\lambda 3 \mathrm{~mm}$. Consequently $S g r A^{*}$ has to be equal or smaller at submm wavelengths than at $\lambda 3 \mathrm{~mm}$.

Once $\nu_{\text {ssa }}$ can be determined, e.g. from broadband variability studies, we can specify the compactness of Sgr A* from its spectral characteristics alone. Arguing that the bulk of the emission at submm and $\mathrm{mm}$ wavelengths comes from two separate components, i.e. requiring $\nu_{\mathrm{ssa}} \sim 100 \mathrm{GHz}$ for the submm component, would imply a source size of only

$$
R \sim 1.5 \cdot 10^{12} \mathrm{~cm} \quad k^{-1 / 17}\left(\frac{F_{\nu_{\max }}}{3.5 \mathrm{Jy}}\right)^{8 / 17}\left(\frac{\nu_{\max }}{10^{12} \mathrm{~Hz}}\right)^{-16 / 51}\left(\frac{\nu_{\mathrm{ssa}}}{100 \mathrm{GHz}}\right)^{-35 / 51}
$$

for $R \sim Z$. This corresponds to $5 R_{\mathrm{g}}\left(=5 \frac{G M_{\mathrm{e}}}{c^{2}}\right)$ of a $2 \cdot 10^{6} M_{\odot}$ black hole and hence to the innermost parts of an accretion disk or the very base of a jet. The fact that the non-thermal spectrum cuts-off towards the IR indicates that the submm regime indeed corresponds to the smallest spatial scale. Do we touch the supermassive black hole at these wavelengths directly?

\subsection{MULTIPLE COMPONENTS}

Although the single, monoenergetic, homogenous blob hypothesis clearly is the simplest description it appears not to be sufficient to explain $\mathrm{Sgr} \mathrm{A}$ and there are several observational indications suggesting a non-homogenous source structure, i.e. 
$\triangleright$ different core sizes at $\lambda 7 \mathrm{~mm}$ and $\lambda 3 \mathrm{~mm}$ (Krichbaum et al. 1994)
$\triangleright$ different variability at radio and submm (Zylka et al. 1995)
$\triangleright$ varying simultaneous spectral indices (Wright \& Backer 1993).

Thus inhomogenous models (with gradients in size, $B$ and $n_{\mathrm{e}}$, e.g. in a jet or an accretion disk) are required to describe Sgr A*.

\section{Spherical Wind Accretion Models}

If we now want to go beyond a mere description of $\operatorname{Sgr~A} \mathrm{A}^{*}$, we have to ask how this source is powered and what the underlying engine producing the radio and $\mathrm{x}$-ray emission actually is? One idea is that if $\mathrm{Sgr} \mathrm{A*}$ is a black hole it should swallow some fraction of the strong stellar winds seen in the GC through Bondi-Hoyle accretion.

The rate of infall depends only on the mass of the black hole and the wind parameters. Once we know the latter we can determine the black hole mass from the estimated accretion rate, which in turn could be derived from the spectrum of $\mathrm{Sgr} \mathrm{A}^{*}$. The general validity of the Bondi-Hoyle accretion (without angular momentum) under these assumptions was recently demonstrated by 3D numerical calculations (Ruffert \& Melia 1994) and the main uncertainties are related to the plasmaphysical effects associated with the infall. It is usually assumed that the magnetic field in the accreted plasma is amplified by compression up to a point where it reaches the equipartition value. Beyond this point the excess magnetic field is assumed to be dissipated and used to heat the plasma. The electron temperature is determined by the equilibrium between heating and cooling via cyclo-synchrotron radiation where one has to consider two domains for the solution of this problem: (1) hot electrons, where the typical electron Lorentz factors are of the order 100-1000 and (2) warm electrons, where the electron Lorentz factor is still close to unity.

The first domain is in a regime where synchrotron emission is important and also very effective. This requires only low accretion rates $(\dot{M} \sim$ $\left.10^{-10} M_{\odot} / y r\right)$ and hence permits only moderately high black hole masses of the order $M_{\bullet} \simeq 10^{3} M_{\odot}$ (Ozernoy 1992). The second domain is in the transition regime between cyclotron and synchrotron radiation, which is less effective than pure synchrotron radiation and hence requires higher accretion rates $\left(\dot{M} \sim 10^{-4} M_{\odot} /\right.$ yr $)$ and a higher black hole mass of the order $M_{\bullet} \simeq 10^{6} M_{\odot}$ (Melia $\left.1992 \& 1994\right)$.

The big advantage of the wind-accretion approach is that it, firstly, appears unavoidable and, secondly, self-consistently ties observable parameters and accretion rate to the mass of the central object. The radio spectrum is well reproduced and initially Melia also was able to account for the $\mathrm{x}$-ray flux. 
On the other hand there are several counter arguments to be considered: Firstly, it is not at all clear that the wind has zero angular momentum, which would diminish the accretion rate and lead to a circularization of the accretion flow further away from the central object. There also could be residual angular momentum in Sgr $\mathrm{A}^{*}$ itself, e.g. because of a fossil accretion disk which could catch the inflow further out, filling a reservoir of rather dense matter instead of directly feeding the black hole. The viscous time scales of such a disk can be very long - up to $10^{7}$ years (Falcke \& Heinrich 1994). More detailed calculation (Falcke \& Melia 1996), show that in fact an intrinsically large angular momentum in the wind is required - a fossil disk alone is not sufficent, as the disk/wind interaction would produce too much NIR emission - to stop (and circularize) the infall at a scale of $10^{16} \mathrm{~cm}$ and to avoid a strong luminosity output.

There are also problems specific to each model. Ozernoy predicts a very compact source which, as shown above, would become self-absorbed already at high radio frequencies and hence requires the presence of other emission components. Melia on the other hand needs a very high accretion rate and, as Ruffert \& Melia (1994) have shown, fluctuations will always lead to the formation of an accretion disk close to the black hole even for the case of initially zero angular momentum. As most of the energy of an accretion disk is produced very close to the black hole it seems impossible to avoid a high luminosity output from this accretion process. The luminosity produced by a Schwarzschild hole $\left(R_{\text {in }}=6 R_{\mathrm{g}}\right)$ is $L_{\text {disk }}=0.8 \cdot 10^{8} L_{\odot} \dot{M} /\left(10^{-4} M_{\odot} / \mathrm{yr}\right)$ and even if the outer disk radius is only two times larger than $R_{\text {in }}, L_{\text {disk }}$ reduces only by a factor 3 . Given the strong limits on the luminosity of Sgr A* of $L_{\text {disk }} \ll 10^{6} L_{\odot}$ it is very unlikely that such a high accretion rate is currently flowing onto the black hole. Finally, the recent SIGMA results (Goldwurm et al. 1994) and especially the ASCA results seem to be in contradiction with the predicted X-ray spectrum of the Melia model.

An alternative to the models mentioned above was proposed by Narayan et al. (1995), who explain the discrepancy between high accretion rate and low luminosity by the effects of an advection dominated disk. In this model more than $99 \%$ of the energy is not radiated but transported through the disk by advection and finally swallowed by the black hole. And in fact it appears as if advection is non-negligible in many accretion disks, but whether indeed such a high fraction of the energy is transported by advection alone is not at all clear. One also has to make sure that not a substantial fraction of the energy is released in the inner parts of the disk and the energy is swallowed quietly by the black hole. Even if an advection dominated disk is not the whole story, it may be an interesting part of it. 


\section{Jet-disk Models}

\subsection{THE BASIC IDEA}

Already in 1980 Reynolds and McKee argued that it is very difficult to confine the synchrotron emitting particles in Sgr A* and proposed a wind or jet model to explain the radio spectrum. Rees (1982) tried to explain Sgr $\mathrm{A}^{*}$ by accretion from the interstellar matter as discussed in the previous section, however, invoking an accretion disk where the synchrotron emission stems from a relativistic electron gas in its inner parts.

We recently suggested to consider a coupled jet disk system for Sgr A* (Falcke et al. 1993a\&b, Falcke \& Biermann 1994\&1995). The basic concept behind this approach - which has also successfully been applied to AGN - is to postulate a fundamental symbiosis between jets and disks around compact objects, i.e. that both always exist and both are energetically important. As the typical escape speed close to a black hole is scale invariant and always a large fraction of $c$ we expect at least mildly relativistic outflows irrespective of the black hole mass. The power of the jet should be mainly governed by the accretion rate.

We extended the classic Blandford \& Königl (1979) jet-emission model by adding mass and energy conservation in a jet-disk system also defining scale invariant parameters for the plasma flow. A more refined model spectrum which includes the effects of adiabatic losses and non-conical jet geometry (see Reynolds 1982) but uses the same basic principles is shown in Fig. 1. Here we also accounted for the presence of a cylindrical region at the base of the jet which we termed 'nozzle', assuming that this is the region where the jet is accelerated and the electrons are injected. Hence the spectrum consists of three regions:

a) the nozzle, dominated by a single, quasi monoenergetic electron distribution producing the submm bump;

b) the jet itself, producing an inverted radio spectrum at $\mathrm{cm}$ wavelengths where the exact spectral index depends on the jet shape and

c) an intermediate region at $m m$ wavelengths where both contribute equally.

The turnover frequencies between those regions depend on the selfabsorption frequency of the submm component and as discussed above on the source size of jet and nozzle. Therefore one expects these parameters to be fixed by either mm-submm VLBI or simultaneous variability studies at $\mathrm{cm}$-submm wavelengths.

The main finding of this kind of model is that size and flux of $\mathrm{Sgr} \mathrm{A}^{*}$ are compatible with it being a radio jet, i.e. the low accretion rate results in a very compact jet but still can yield a 1 Jy source. Although the overall power of the jet is fairly low due to the low accretion rate, the ratio between 


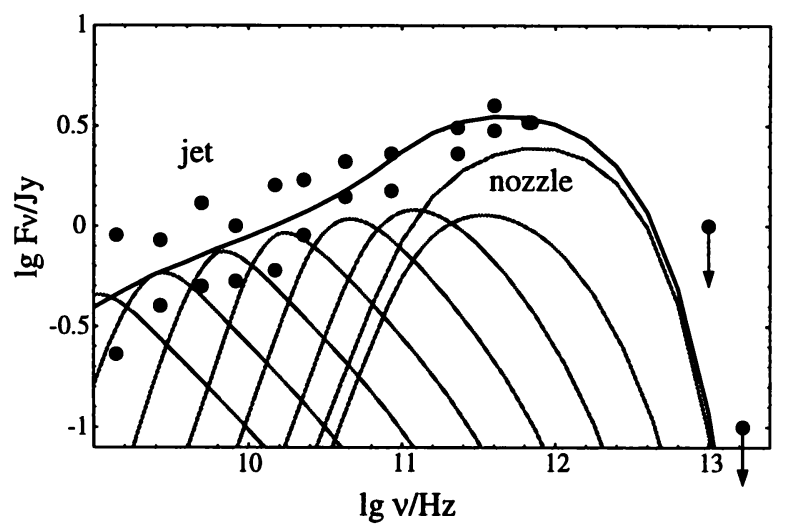

Figure 1. Model spectrum for jet and nozzle coupled to an accretion disk in Sgr A*. Parameters are: $R_{\text {nozz }}=3 \cdot 10^{11} \mathrm{~cm}, Z_{\mathrm{nozz}}=4.25 \cdot 10^{12} \mathrm{~cm}, \gamma_{\mathrm{e}}=70, q_{\mathrm{j} / 1}=0.35$, $L_{\text {disk }}=10^{39} \mathrm{erg} / \mathrm{sec}, x_{\mathrm{e}}=1, \gamma_{\mathrm{j}}=2, i=60^{\circ}$ (see Falcke \& Biermann 1995). We included adiabatic losses, a nozzle where electrons are injected monoenergetically and a jet with shape $R_{\mathrm{j}}=R_{\text {nozz }}+\left(Z_{\mathrm{j}} / Z_{\text {nozz }}\right)^{0.55} / \mathcal{M}$ slowly reaccelerating electrons into a $p=2.5$ powerlaw.

jet power $Q_{\text {jet }}$ and $L_{\text {disk }}$ appeared relatively high $(\sim 0.3-1)$. This can easily be checked by crudely estimating the magnetic luminosity of Sgr $\mathrm{A}^{*}$ which is $L_{\mathrm{B}} \sim 0.125(10 \mathrm{G})^{2}\left(10^{13} \mathrm{~cm}\right)^{2} c \sim 10^{4} L_{\odot}$. Now, one only has to remember that the total jet power including relativistic particles and kinetic energy is at least 3-4 times higher and that probably $L_{\text {disk }} \leq 10^{5} L_{\odot}$.

\subsection{THE AGN CONNECTION - THE CASE FOR HADRONIC CASCADES}

We found that the same kind of model can not only explain Sgr A* but also the jets in AGN and even account for the tight UV-radio correlation in radio weak quasars (Falcke et al. 1995b).

Once more the limits imposed by the accretion disk played a crucial rôle. Again one infers injection of relativistic electrons (positrons) at high energies above $\gamma_{e}=100$ for radio loud jets and we argued that perhaps the difference between radio loud and radio weak quasars could be understood by the lack of this efficient injection mechanism in radio weak quasars (Falcke, Gopal-Krishna, Biermann 1995a). Anyway, the similarity of the high electron Lorentz factors found (directly) in Sgr A* and (indirectly) in AGN is more than striking. Hence we suggested that this typical Lorentz factor has a basic physical reason, namely the $\pi$-decay following hadronic cascades initiated by $p p$-collisions between relativistic protons in the jet and thermal protons surrounding the jet. Because of the high rest mass of the $\pi$ the secondary pairs produced in the cascade will have a characteristic energy of $>35 \mathrm{MeV}\left(\gamma_{\mathrm{e}}>70\right)$ (see also Biermann et al. 1995). Jets interacting with 
a dense medium can inject additional high energy secondary electrons and become radio loud, while those which do not interact remain radio weak with only primary electrons injected at thermal energies - in this respect Sgr A* is radio loud. The latter remains true if one extends the $L_{\text {disk-radio }}$ correlation of AGN to lower luminosities and includes nearby Galaxies with detected radio cores and even stellar mass black holes (Falcke 1994, Falcke $\&$ Biermann 1996): again one finds something like a radio loud/radio weak dichotomy, smoothly connecting to AGN, with Sgr A* beeing fairly loud.

Where exactly those $p p$-collisions might occur in $\mathrm{Sgr} \mathrm{A}^{*}$ is still uncertain: they may happen in an interaction zone between the jet and infalling wind or the dense absorbing material discovered by ROSAT (Predehl \& Trümper 1994), but even the disk or the wind (Mastichiadis \& Ozernoy 1994 ) itself could be a site for proton (shock-)acceleration. If $p p$-collisions are the dominant cooling process for relativistic protons being accelerated in a dense medium this would naturally yield monoenergetic secondary electrons. Below the $\pi$-production threshhold at $140 \mathrm{MeV} p p$-collisions are inelastic and neither produce secondaries nor lead to cooling of the protons. Once the protons are accelerated above the threshhold energy for $\pi$-production, they will instantaneously cool by $p p$-collisions until they fall below the threshhold energy thus bouncing back and forth around this energy. The resulting secondary electrons would be injected in a narrow energy interval at roughly $1 / 4$ of the the threshold energy yielding $\gamma_{\mathrm{e}} \geq 70$.

\section{Summary}

Considering the dynamical and spectral evidences I have no doubt that indeed Sgr A* is the very center of the Galaxy and hence will have the coordinates $l_{3}=0$ and $b_{3}=0$ after the next revision of the galactic coordinate system (to be proposed at a future IAU assembly). Current observational data constrain models for $\mathrm{Sgr} \mathrm{A}^{*}$ already much stronger than for any other galactic nucleus - we will never get closer to a supermassive black hole. Although many question are still disputed, there is now some consensus that $\mathrm{Sgr} \mathrm{A}^{*}$ is currently put on a starvation diet - despite its high mass and strong stellar winds in the surroundings. A coupled jet/disk system can explain the spectral and structural characteristics of Sgr A* quite well and its smallest source size is close to the typical size of a black hole of mass $M_{\bullet} \sim 10^{6}$, while the typical electron Lorentz factor of $\gamma_{\mathrm{e}} \sim 100$ may be indicative of hadronic cascades. Crucial future experiments will be simultaneous variability studies and mm-submm VLBI observations. Both, however, will require joined efforts to face a single but promising challenge - understanding $\operatorname{Sgr} A^{*}$. 


\section{References}

Backer D.C., Zensus J.A., Kellermann K.I. et al. 1993, Science 262, 1414

Biermann P.L., Strom R., Falcke H. 1995, A\&A 302, 429

Blandford R.D., Königl A. 1979, ApJ 232, 34

Duschl W.J., Lesch H. 1994, A\&A 286, 431

Eckart, A., Genzel, R., Krabbe, A., Hofmann, R., van der Werf, P. P., Drapatz, S. 1992, Nat 355, 526

Eckart, A., Genzel, R., Hofmann, R., Sams, B.J., Tacconi-Garman, L.E. 1993, ApJ 407, L77

Eckart, A., Genzel, R., Hofmann, R., Sams, B.J., \& Tacconi-Garman, L.E. 1995, ApJ 445, L23

Falcke H. 1994, PhD thesis, RFW Universität Bonn

Falcke H., Biermann P. L. 1994, in "Mass Transfer Induced Activity in Galaxies", Shlosman, I. (ed.), Cambridge University Press, p. 44

Falcke H., Biermann P.L. 1995, A\&A 293, 665

Falcke H., Heinrich O. 1994, A\&A 292, 430

Falcke H., Biermann P.L. 1996, A\&A in press

Falcke H., Melia F. 1996, ApJ submitted

Falcke H., Biermann P. L., Duschl W. J., Mezger P. G. 1993a, A\&A 270, 102

Falcke H., Mannheim K., Biermann P. L. 1993b, A\&A 278, L1

Falcke H., Gopal-Krishna Biermann P. L. 1995a, A\&A 298, 395

Falcke H., Malkan M., Biermann P.L. 1995b, A\&A 298, 375

Genzel R., Townes R., 1987, ARA\&A 25, 377

Goldwurm A. et al. 1994, Nat 371, 589

Gwinn C.R., Danen R., Middleditch J., Ozernoy L., Tran T. 1991, ApJ 381, L43

Haller J., Rieke, M., Rieke, G., Tamblyn, P. Close, L. \& Melia, F. 1995, Ap.J., in press

Krabbe A., Genzel R., Drapatz S., Rotaciuc V., 1991, ApJ 382, L19

Krichbaum T.P., Zensus J.A., Witzel A., et al. 1993, A\&A 274, L37

Krichbaum T.P., Schalinski C.J., Witzel A., Standke K., Graham D.A., and Zensus J.A. 1994, in "The Nuclei of Normal Galaxies: Lessons from the Galactic Center", eds. Genzel R. \& Harris A.I., Kluwer, Dordrecht

Kundt W. 1990, A\&ASS 172, 130

Koyama, K. 1994, in: New Horizon of X-ray Astronomy - first results from ASCA, Universal Academy Press, p. 181

Maeda Y., Koyama K., Sakano M., Takeshima T., Yamauchi S. 1996, to appear in PASJ

Mastichiadis A., Ozernoy L. 1994, ApJ 426, 599

Mattox J.R., Bertsch D.L., Fichtel C.E. 1992, BAAS 24, 1296

Melia F. 1992, ApJ 387, L25

Melia F. 1994, ApJ 426, 677

Narayan R., Yi I., Mahadevan R. 1995, Nat 374, 623

Ozernoy L. 1992, in AIP Conf. Proc. 254, Testing the AGN Paradigm, ed. S.S. Holt et al., New York, p. 40,44

Predehl P., and Trümper J. 1994, A\&A 290, L29

Rees M. 1982, in: "The Galactic Center", AIP Conf. 83, eds. Riegler \& Blandford, p. 166

Reynolds S.P. 1982, ApJ 256, 13

Reynolds S.P., McKee C.F. 1980, ApJ 239, 893

Rogers A.E.E., Doeleman S., Wright M.C.H. et al. 1994, ApJ 434, L59

Ruffert M., Melia F. 1994, A\&A 288, L29

Sunyaev R. et al. 1991, ApJ 383, L49

Wright M.C.H., Backer D.C. 1993, ApJ 417, 560

Zylka R., Mezger P.G., Lesch H. 1992, A\&A 261, 119

Zylka R., Mezger P.G., Ward-Thomson D., Duschl W., Lesch H. 1995, A\&A 297, 83 


\section{DISCUSSION}

C. Townes: There are strong stellar winds in the GC. How can you avoid a high mass inflow - like in the Melia model - if Sgr A* is a supermassive black hole?

Falcke: Given the bolometric luminosity constraints for UV and X-rays, I think that accretion rates as high as $10^{-4} M_{\odot} / \mathrm{yr}$ are already ruled out by observations. Why Sgr A* does not accrete more matter remains a mystery. Obviously we do not yet understand angular momentum distribution and transport in the inner $0.1 \mathrm{pc}$ around Sgr $\mathrm{A}^{*}$. On the other hand I can not completely rule out that $\mathrm{Sgr} \mathrm{A}^{*}$ is less massive than we think it is.

C. Townes: Does the mass of Sgr A* play an important rôle in your models.

Falcke: Not really. Besides the dynamical estimates, only the fact that the limits for the submm source size - giving the smallest scale - are so close to what we expect for a $10^{6} M_{\odot}$ black hole seems very suspicious. A sign for a low mass black hole would be thermal x-ray emission from an accretion disk and heating of the ambient gas.

T. Hasegawa: Do you have any comments on the accretion history of the black hole? Do we see any signs of episodes of higher accretion rate in the past, or has it been starving from the very beginning of its formation?

Falcke: There is a weak feature - the so called GC spur (Sofue, Reich \& Reich 1989, ApJ 341, L47) - which could be the smoke trail of past jet activity. A single giant molecular can turn the GC into a Seyfert nucleus at any time and this could have happened already in the past. If the winds of the surrounding stars really are captured by a fossil accretion disk around Sgr $\mathrm{A}^{*}$ and are stored in a close orbit than this could also lead to recurrent activity on a time scale of $10^{5}-10^{7}$ years. 